\title{
Cloning and expression of a novel component of the CAP superfamily enhanced in the inflammatory response to LPS of the ascidian Ciona intestinalis
}

\author{
Angela Bonura • Aiti Vizzini • Giuseppina Salerno • \\ Daniela Parrinello • Nicolò Parrinello • Valeria Longo • \\ Giovanna Montana $\cdot$ Paolo Colombo
}

Received: 20 July 2010 / Accepted: 5 October 2010

(C) Springer-Verlag 2010

\begin{abstract}
The CAP superfamily is a group of proteins that have been linked to several biological functions such as reproduction, cancer, and immune defense. A differential screening between lipopolysaccharide (LPS)-challenged and naive Ciona intestinalis has been performed to identify LPS-induced genes. This strategy has allowed the isolation of a full-length 1471-bp cDNA encoding for a 413-aminoacid protein (CiCAP). In silico analysis has shown that this polypeptide displays a modular structure with similarities to vertebrate CAP-superfamily proteins and to a collagenbinding adhesin of Streptococcus mutans. Domain organization analysis and alignment of CiCAP to other vertebrate CAP proteins have revealed a novel structure suggesting that this protein originated from a common ancestor gene that gave rise to many subfamilies of mosaic proteins with novel functions. Quantitative mRNA expression performed by real-time polymerase chain reaction analysis has demonstrated that this gene is rapidly activated in the pharynx of $C$. intestinalis a few hours after LPS injection. Moreover, in situ hybridization has shown that CiCAP mRNA is highly expressed by hemocytes with large granules contained inside the pharynx vessels. Thus, CiCAP represents a protein with novel structural domains involved in ascidian immune responses.
\end{abstract}

\footnotetext{
A. Bonura $\cdot$ V. Longo $\cdot$ G. Montana $\cdot$ P. Colombo $(\triangle)$ Istituto di Biomedicina ed Immunologia Molecolare "Alberto Monroy" del Consiglio Nazionale delle Ricerche, Via Ugo La Malfa 153, 90146 Palermo, Italy

e-mail: paolo.colombo@ibim.cnr.it

A. Vizzini $\cdot$ G. Salerno $\cdot$ D. Parrinello $\cdot$ N. Parrinello Dipartimento di Biologia Animale, Università di Palermo, Via Archirafi 18,

Palermo, Italy
}

Keywords Innate immune system - Differiantial display CAP protein $\cdot$ Molecular biology $\cdot$ Ciona intestinalis (Tunicata)

\section{Introduction}

The CAP superfamily represents a group of diverse proteins that have been isolated in several species ranging from Saccaromyces cerevisiae to mammals. Within mammals, CAP proteins have been identified in several tissues in which they perform endocrine or paracrine functions linked to several cellular processes including reproduction, development, immune functions, and several pathologies including cancer, nerve damage, pancreatitis, and heart failure (for a review, see Gibbs et al. 2008). The CAP nomenclature is derived from the three major groups of proteins originally identified, viz., the cysteine-rich secretory proteins (CRISPs), antigen $5(\mathrm{Ag} 5)$ and pathogenesis-related 1 protein families (Pr-1), suggesting a functional link between the plant and the animal immune system (Szyperski et al. 1998). The CAP superfamily is characterized by several signature sequences that are usually present in a small disulfide-bond stabilized and structurally conserved $17-$ to $21-\mathrm{kDa}$ domain that has been referred to as the CAP domain. Although a large number of proteins within the superfamily contain a CAP domain in isolation (e.g., the Ag5 and Pr-1 proteins found in insects and plants), many others contain additional Cterminal extensions that can impart diversity of biological functions. Most of them have a short C-terminal extension containing four cysteines, which has been referred to as a Hinge region (Guo et al. 2005). Additional domains are sometimes entirely unique and are observed only in association with the CAP domain, such as the ion channel 
regulator at the $\mathrm{C}$ terminus of the CRISPs (Gibbs et al. 2006); some others display homology to domains of diverse protein families, such as the C-type lectin domain (Zelensky and Gready 2005) and the LCCL domain (Trexler et al. 2000). Among non-mammalian proteins, various CAP proteins have been found in Drosophila (for a review, see Kovalick and Griffin 2005), Xenopus, Heloderma horridum, and Conus textile (for a review, see Yamazaki and Morita 2004). CAP proteins have been identified in several species of both free-living (Caenorhabditis elegans) and parasitic nematodes. These proteins are produced at the crucial early stage of parasitic invasion to evade the host immune system and facilitate feeding (Del Valle et al. 2003; Hawdon and Hotez 1996; Moyle et al. 1994).

Ascidians (subphylum Tunicata) are hermaphroditic invertebrates belonging to the same phylum of vertebrates (Chordata) (Delsuc et al. 2006; Swalla et al. 2000) and occupying an intermediary phylogenetic position between invertebrates and vertebrates (Azumi et al. 2003). For this reason, they represent an intriguing model for studying the evolution of the innate immune system. In particular, the inflammatory reaction in the body wall of $C$. intestinalis (Parrinello et al. 1984, 1990) is a well-established model for the analysis of inducible host defence molecules of the innate immune system (Bonura et al. 2009; Cammarata et al. 2007, 2008; Parrinello et al. 2008; Vizzini et al. 2008). The pharynx is the main hemopoietic organ in which circulating hemocyte populations are renewed, and it can be challenged by inoculating inflammatory agents. CAP proteins have previously been isolated from ascidians. Halocynthia roretzi (sea squirt) presents two CAP proteins (HrUrabin and HrUrabin-long) (Urayama et al. 2008) with a high percentage of homology to glioma pathogenesisrelated proteins (GLIPR1L1 and GLIPR1L2) respectively, which are expressed within testes (Ren et al. 2006) and on sperms (Gibbs et al. 2010), representing the first CAP proteins identified within invertebrate sperm. These proteins seem to play a role as adhesion molecules involved in the negative selection against "self" oocytes. However, little is known about other ascidian CAP proteins and their involvement in biological functions apart from the control of self-fertilization.

In the present paper, we report a $C$. intestinalis CAP (CiCAP) cDNA that exhibits a novel modular structure. CiCAP shows a $\mathrm{NH}_{2}$ terminal domain with a high level of homology to the vertebrate CAP superfamily, and a $\mathrm{COOH}$ terminal domain highly homologous to the collagen-binding adhesin of Streptococcus mutans. Real-time polymerase chain reaction (PCR) analyses and in situ hybridization (ISH) has revealed that inoculation of lipopolysaccharide (LPS) into the ascidian pharynx up-regulates the CiCAP gene suggesting that the CiCAP gene is involved in the innate immune response of $C$. intestinalis.

\section{Materials and methods}

Tunicates

Ascidians were collected from Sciacca Harbor (Sicily, Italy), maintained in tanks with aerated sea water at $15^{\circ} \mathrm{C}$, and fed every second day with a marine invertebrate diet (coraliquid) (Sera Heinsberg, Germany). C. intestinalis were kept in facilities compatible with their normal requirements and under conditions that minimize stress, by following guidelines for marine invertebrate maintenance.

\section{LPS injection}

LPS (Escherichia coli 055:B5, LPS; Sigma-Aldrich, Germany) solution was prepared in sterile marine solution (MS:12 mM $\mathrm{CaCl}_{2} \times 6 \mathrm{H}_{2} \mathrm{O}, 11 \mathrm{mM} \mathrm{KCl}, 26 \mathrm{mM} \mathrm{MgCl}_{2} \times 6 \mathrm{H}_{2} \mathrm{O}, 43 \mathrm{mM}$ TRIS HCl, $0.4 \mathrm{M} \mathrm{NaCl}$, pH 8.0). LPS solution (100 $\mu \mathrm{g}$ LPS in $100 \mu \mathrm{l}$ MS per animal) was injected through the tunic into the body wall (pharyngeal region) at the mid-portion referred to the longitudinal axis. Ascidians, both untreated and injected with MS only $(100 \mu \mathrm{l})$, were used as negative controls.

\section{Total RNA extraction and poly(A) ${ }^{+}$purification}

Ascidian tissue fragments $(200 \mathrm{mg})$ explanted at various times (from 1 to $48 \mathrm{~h}$ ) were immediately soaked in RNAlater Tissue collection (Ambion, Austin, Tex., USA) and stored at $-80^{\circ} \mathrm{C}$. Total RNA extraction was performed by using an RNAqueous-Midi Kit purification system (Ambion).

Poly $(\mathrm{A})^{+}$RNA was prepared from control and injected animals $(1 \mathrm{~h})$ by using an Illustra mRNA Purification Kit (GE Healthcare, UK) according to the manufacturer's instructions. The RNA quality was assessed by agarose gel electrophoresis, and the RNA concentration and purity were determined by measuring the absorbance at 260 and $280 \mathrm{~nm}$ (data not shown).

\section{Subtractive hybridization}

Subtractive hybridization was performed with the PCRSelect cDNA Subtraction Kit (Clontech Laboratories, USA) according to the manufacturer's instructions. This strategy is based on a PCR-based method for the selective amplification of differentially expressed sequences, thereby allowing the isolation of transcript from activated tissues (pharynx). Briefly, $2 \mu \mathrm{g}$ poly $(\mathrm{A})^{+}$RNA from non-injected (driver) and injected (tester) animals were retro-transcribed. The tester and driver cDNAs were digested with the restriction enzyme $R s a$ I to yield blunt ends. The tester cDNA was then subdivided into two parts, and each was ligated with a different cDNA adaptor (ADAPTOR 1: 5'-CTAATAC GACTCACTATAGGGCTCGAGCGGCCGCCCGGG 
CAGGT-3'; ADAPTOR 2: 5'-CTAATACGACTCACTA TAGGGCAGCGTGGTCGCGGCCGAGGT-3'). The ends of the adaptor did not contain a phosphate group, and so only one strand of each adaptor attached to the $5^{\prime}$ ends of the cDNA. Two hybridizations were then performed. In the first run, an excess of driver was added to each sample of tester. The samples were then heat-denatured and allowed to anneal. In the second run of hybridization, the two primary hybridization samples were mixed together without denaturing to allow the subtracted single-strand tester cDNAs to re-associate. These new hybrids were molecules that had different ends but that corresponded to the sequences of the two adaptors. After filling in the ends by DNA polymerase, the differentially expressed sequences displayed different annealing sites for the nested primers on their $5^{\prime}$ and $3^{\prime}$ ends. The entire population of molecules was then subjected to PCR in order to amplify the desired differentially expressed sequences by using the following primers (Nested PCR Primer 1: 5'-TCGAGCGGCCGCCC GGGCAGGT-3'; Nested PCR Primer 2: 5'-AGCGTGG TCGCGGCCGAGGT- $\left.3^{\prime}\right)$ and PCR conditions $\left(94^{\circ} \mathrm{C}\right.$ for $30 \mathrm{~s}, 68^{\circ} \mathrm{C}$ for $30 \mathrm{~s}, 72^{\circ} \mathrm{C}$ for $90 \mathrm{~s} ; 12$ cycles).

\section{Cloning and sequence analysis}

Differentially expressed cDNAs were cloned in the pCR4TOPO vector (Invitrogen, USA) and sequenced. Sequence analysis showed a cDNA fragment of 94 nucleotides. Similarity searches performed by using the FASTA algorithm (http://www.ebi.ac.uk/Tools/fasta/) showed relevant homology to some expressed sequence tag clones from mature adult $C$. intestinalis (data not shown). The fulllength sequence of the cDNA clone was obtained by using the GeneRacer kit (Invitrogen, USA). The kit ensures the amplification of only full-length transcript via elimination of truncated messages from the amplification process. 5' Rapid amplification of cDNA ends (RACE) was performed by PCR $\left(94^{\circ} \mathrm{C}\right.$ for $60 \mathrm{~s}, 52^{\circ} \mathrm{C}$ for $60 \mathrm{~s}, 72^{\circ} \mathrm{C}$ for $60 \mathrm{~s} ; 30$ cycles) with the CiCAP $3^{\prime}$ RACE-specific oligonucleotide 5'-AAGGTTCTGCCAGTTCCTC-3' (see Fig. 1 for details) and the GeneRacer 5'-oligonucleotide, viz., 5'- CGACTGG AGCACGAGGACACTGA-3'. This reaction was diluted 1:100, and a nested PCR was performed with the GeneRacer 5' nested-oligonucleotide (5'-GGACACTGA CATGGACTGAAGGAGTA-3') and the CiCAP 3' nested specific oligonucleotide (5'-CTGTGGACGACATTGT-3').

3' RACE was performed with the Ciona CiCAP 5'specific oligonucleotide (5'-ACAATGTCGTCCACAG-3') and the GeneRacer $3^{\prime}$ oligonucleotide (3'-GCAATGCA TCGCATAGCAACTGTCG-5'). PCR products were diluted 1:100 and re-amplified with the CiCAP $5^{\prime}$ specific oligonucleotide and the GeneRacer 3' nested oligonucleotide (3'-GTGACAGTACGGCAATGCATCGC-5'; see Fig. 1 for details). Fragments were purified, ligated in the pCR4TOPO vector (Invitrogen, USA), and sequenced.

Sequence and phylogenetic analysis

Similarity searches were performed by using the FASTA program (http://www.ebi.ac.uk/Tools/fasta/). They showed a high degree of homology of CiCAP to several CAPsuperfamily proteins from vertebrates and invertebrates. Multiple alignments were accomplished with the Clustal W program (Thompson et al. 1994). A phylogenetic tree was constructed by the neighbor-joining method, and 1000 bootstrap replicates were performed. The respective accession numbers are as follows: human cysteine-rich secretory protein LCCL domain-containing 2(CRLD2), Q9H0B8; Mus musculus cysteine-rich secretory protein LCCL domain-containing 2 (CRLD2), Q8BZQ2; Xenopus laevis cysteine-rich secretory protein LCCL domain-containing 2 (CRLD2), Q4V9Y5; human GLIPR1, P48060; Bos taurus GLIPR1, Q32LB5; Mus musculus GLIPR1, Q9CWG1; Osmerus mordax GLIPR1, C1BLT0; human CRISP2, P16562; human GLIPR2, NP071738; Mus musculus GLIPR2, NP081726; Salmo salar GLIPR1, ACI33913; Xenopus laevis CRISP, NP001082594; Schistosoma mansoni CRISP, XP002578074; Mus musculus peptidase inhibitor 15 (PI15), NP444421; human PI15, AAI26293; Danio rerio PI15, NP_001153449; human PI16, AAH35634; Mus musculus PI16, CH466606.1; Bos taurus PI16, Q58D34; Polistes fuscatus Ag5, P35780; Vespula vulgaris Ag5, CAB42887.

\section{Real-time PCR analysis}

Tissue expression of the CiCAP gene was studied in four distinct LPS-challenged ascidians by real-time PCR with the Sybr-Green method. Primers were designed by using Custom Primers OligoPerfect Designers software (https:// tools.invitrogen.com/) and synthesized commercially (Eurofins MWG Operon, Ebersberg, Germany). Real-time PCR analysis was performed on the Applied Biosystems 7500 real-time PCR system. Tissue expression was performed in a $25-\mu 1$ PCR containing $2 \mu \mathrm{l}$ cDNA converted from $250 \mathrm{ng}$ total RNA, $300 \mathrm{nM}$ CiCAP $1 \mathrm{~F}$ forward (5'GTATCTCCGCGAAGAGTTGG-3') and CiCAP 1R reverse primers ( $5^{\prime}$-TCGGTATAACGTCGCCTCT-3'), $300 \mathrm{nM}$ actin forward (5'-TGATGTTGCCGCACTCGTA-3') and actine reverse (5'-TCGACAATGGATCCGGT-3') primers, and $12.5 \mu 1$ Power Sybr-Green PCR Master Mix (Applied Biosystems). The 50 cycles of the two-step PCR program consisted of initial polymerase activation for $3 \mathrm{~min}$ at $95^{\circ} \mathrm{C}$ followed by a denaturing step at $95^{\circ} \mathrm{C}$ for $15 \mathrm{~s}$, and then annealing/extension was carried out at $60^{\circ} \mathrm{C}$ for $45 \mathrm{~s}$ when the fluorescent signal was detected. Each set of samples was run 
61 taagactgaacattgctcaATGGATTCTAAACTGCATGTTACTTTCGCTGTGTTGCTTTT

121 ACTGCTCGCTTCTTTTACAAATGCTATAGAATGGTCCGAGTCCATAAATGCTGAAGAAGC

181 ACACCAGCGGTCGAAGCGCAGCCCTGACCAGCTGCTAGAGACAGTCGTTTTAACCCAGGC GGAAAAGGATCAAATCGTGGCCTTGCACAATAAGTTCCGTTCTGATGAAATGGCGTCAAA

301 TATGCTAGCAATGTCCTGGGATAACCAAATCGCGAATTTCGCATCATCCTATATATCAAA

361 TTGTGAGTTTTCGCACAGTAGTTCGGCGGAGCGGAGCCGGCATCCCATATTTAGTTATTT

481 CCGTGTCAATCAATTGTGGTACGATGAAATAAATGACTATACGTACAGTATTTTCACGTG

541 TGCTGCTGGCAAAGCATGCGGCCATTACACTCAACAAGTTTGGGCTAGTACCTACAAGAT 600

601 AGGTTGCGGAGCTGCATACTGCGCTCGTGGGAACGGTGGTTCGGGATATCAACTCATGGT 660

661 AGCGTGTCAATACGGACCTGGTGGCAATCTCCTTAATACGAATGTATCTCCGCGAAGAGT 720

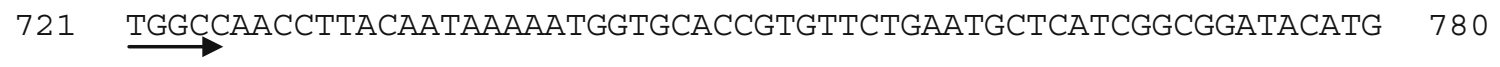

781 TGTTAACAATCTGTGCATGAATCTTGCGAGAGGCGACGTTATACCGACCAACAGTACACC 840

841 AACAACCACAAGTACACCAACAACTACAAGTACACCAACAACTACAAGTACACCAACAAC 900

901 TACAAGTACACCAACAACTACAAGTACACCAACAACTACAAGTACACCAACAACTAGCAG 960

961 TACACCAACAACTACAAGTACACCAACAACTACAAGTACACCAACAACTACAAGTACACC 1020

1021 AACAACTAGCAGTACACCAACAACTAGCAGTGCACCAACAACTAGCAGTACACCAACAAC 1080

1081 CAGCAGTACCTCTCCACCTACAACGAGCTCAGGGCCACCTTCCACAACTGAAAGACCAAG 1140

1141 CAGTACACCCCCGCCTACAATGTCGTCCACAGCACCCACCAGCACCTCAGCACCGACCAC 1200

1201 TGACCCTACGACCGGTCAATGCCAGGCGATTCAAGAGTACAAAGAAGCTTTAGCAGCATA 1260 CAP 3'UTR forward

1261 CCAGACGGAACTGAGGAACTGGCAGAACCTTTTCAATGCTTGGTTGGCTGCACAGCCGTG 1320

1321 Aaaacatacaaaaccgcacttatacaattcgaaagatttgcacacatttactgcagtcgt 1380

1381 cattttttgcttaatataacatctgtatacccgatatagcttatcaaatataatagttg 1440

1441 tactgcacagaaaaaaaaaaaaaaaaaaa 1471 CAP 3'UTR reverse

Fig. 1 Nucleotide sequence of the full-length CiCAP. 5'- and 3'Untranslated regions (UTR) are given in lower case letters. Upper case letters indicate the coding region with the first ATG and the stop codon highlighted in bold letters (arrows oligonucleotide used for cloning procedures). The region from nucleotide 1320 to nucleotide 1450 is the probe used for the in situ hybridization (ISH) assay (CAP 3'UTR forward oligonucleotide vs CAP 3'UTR reverse oligonucleotide) 
three times, and each plate contained quadruplicate cDNA samples and negative controls. The specificity of amplification was tested by real-time PCR melting analysis. To obtain sample quantification, the $2^{-\Delta \Delta \mathrm{Ct}}$ method was used, and the relative changes in gene expression were analyzed as described in the Applied Biosystems Use Bulletin N.2 (P/N 4303859). The amount of CiCAP transcript from the various tissues was normalized to actin in order to compensate for variations in input RNA amounts. Relative CiCAP expression was determined by dividing the normalized value of the target gene in each tissue by the normalized value obtained from the untreated tissue.

Body wall explant preparation and histology

The ascidian body wall consists of the tunic, connective tissue with lacunae of the open circulatory system, muscles, and the large pharynx sac provided with vessels containing hemocytes. The tunic surface was cleaned with ethyl alcohol, and body wall fragments $(200 \mathrm{mg})$, containing both tunic and pharynx tissue, were excised from the injection site at various times after injection (1-48 h).

For ISH studies, body wall fragments were fixed in Bouin's fluid (picric acid solution:formaldehyde:acetic acid 15:5:1) for $24 \mathrm{~h}$, paraffin embedded, and serially cut at $6 \mu \mathrm{m}$ (Leica RM2035 microtome, Solms, Germany).

\section{ISH assay}

To examine tissue excised from the inflamed body wall, ISH was carried out with digoxigenin-11-UTP-labeled riboprobes $(1 \mu \mathrm{g} / \mathrm{ml}$ final concentration). The CiCAP probe was generated by PCR-amplifying a cDNA fragment of $148 \mathrm{bp}$ covering the $3^{\prime}$ untranslated region from nt 1302 to nt 1450 of the isolated cDNA with the CAP 3'UTR forward (5'-GGCTGCACAGCCGTGA-3') and the CAP 3'UTR reverse (5'-CTGTGCAGTACAACT-3') oligonucleotides (see Fig. 1). The DNA fragment was cloned in the pCR4TOPO vector (Invitrogen, USA). The CiCAP digoxigenin11-UTP-labeled riboprobes were produced according to the manufacturer's instructions (Roche Diagnostics). The re-hydrated histological sections were digested with proteinase $\mathrm{K}(10 \mu \mathrm{g} / \mathrm{ml})$ in phosphate-buffered saline (PBS) for $5 \mathrm{~min}$, washed with PBS plus Triton X-100 (PBS-T), and treated for hybridization with $50 \%$ formamide, $5 \times \mathrm{SSC}(1 \times \mathrm{SSC}: 0.15 \mathrm{M} \mathrm{NaCl} / 0.015 \mathrm{M}$ sodium citrate, $\mathrm{pH} 7)$, $50 \mu \mathrm{g} / \mathrm{ml}$ heparin, $500 \mu \mathrm{g} / \mathrm{ml}$ yeast tRNA, and $0.1 \%$ Tween 20 , at $37^{\circ} \mathrm{C}$ overnight. After exhaustive washes in PBS-T and $4 \times \mathrm{SSC}$ (twice for $10 \mathrm{~min}$ ), the sections were incubated for $1 \mathrm{~h}$ with anti-digoxigenin-Fab-AP conjugate (Roche Diagnostics) diluted 1:500 and washed in PBS-T. Finally, the sections were incubated in the 5-bromo-4chloro-3-indolyl phosphate/nitro-blue tetrazolium liquid substrate system (Sigma-Aldrich, Germany). Color development was stopped after $30 \mathrm{~min}$ at room temperature. Four independent ascidians (untreated or inoculated with MS) were used as control animals.

\section{Statistical methods}

Student's $t$-test was used to estimate statistical significance. Multiple comparisons were performed with a one-way analysis of variance, and the different groups were compared by using Tukey's $t$-test. Standard deviations were calculated from four experiments. $P<0.01$ was considered as being statistically significant.

\section{Results}

CiCAP is a member of the CAP-superfamily protein

A PCR-based subtractive hybridization strategy from the naive and inflamed pharynx of $C$. intestinalis allowed the isolation of a new LPS-induced form of CAP protein (CiCAP). 5' and 3' RACE was used to identify full-length mRNAs, and sequence analysis showed a 1471-nt cDNA with 5' (79 nucleotides) and 3' (150 nucleotides) untranslated regions (see Fig. 1 for details). A BLAST search in the Ensembl genome browser identified the predicted gene ENSCING00000004464 localized on chromosome 8q: $2,547,479-2,550,637$. The cDNA encodes a 413-aminoacid protein with a deduced molecular size of 43895 Dalton (Fig. 2). In particular, in silico analysis showed that the CiCAP contained a 21-amino-acid-long signal peptide and displayed several CAP-superfamily domains identified in the 61-240 tract: the CiCAP3 motif [HNKFR] (63-67), the CiCAP4 motif [GENL] (115-118), the CiCAP1 motif [GHYTQQVWASTYKIGC] (161-176), and the CiCAP2 motif [MVACQYGPGGNL] (193-204). In addition, a GLIPR1 motif $\left[\mathrm{CX}_{2} \mathrm{CX}_{5} \mathrm{CX}_{4} \mathrm{CX}\right]$ was present in the 225240 region. Sequence analysis showed a highly conserved "PY" dipeptide (amino acids 117-118) characteristic of the CAP-superfamily protein. Finally, the CiCAP C-terminal domain contained several threonine residues considered to be putative glycosylation sites in the T-N-TTT-N-TP repeat sequences (Fig. 2).

Molecular phylogenetic analysis

A similarity search with the FASTA algorithm showed a significant homology of CiCAP with vertebrate CAPsuperfamily proteins. In particular, the amino-acid sequence $1-240$ showed the highest similarity $(61.4 \%)$ and identity (39.1\%) to human GLIPR1 (P48060; Fig. 3a), and the amino-acid sequence 240-413 showed a significant similarity 
Fig. 2 cDNA-deduced aminoacid sequence of the CiCAP protein. Locations of putative structural domains are based on the Predict Protein algorithm. The putative transmembrane domain is indicated in italics. Asterisks show the ten characteristic CAP-family cysteine residues, doubleunderlined amino acids the conserved "PY" CAP dipeptide, boxed amino acids the CAP domains and the GLIPR1 domain $\left[\mathrm{CX}_{2} \mathrm{CX}_{5} \mathrm{CX}_{4} \mathrm{CX}\right]$. The conserved amino acids within the domains are in bold. The underlined threonine residues are the putative glycosylation sites in the T-N-TTT-N-TP repeat sequences
1

Transmembrane domain

50 MDSKLHVTFA VLLLLLASFT NAIEWSESIN AEEAHQRSKR SPDQLLETVV

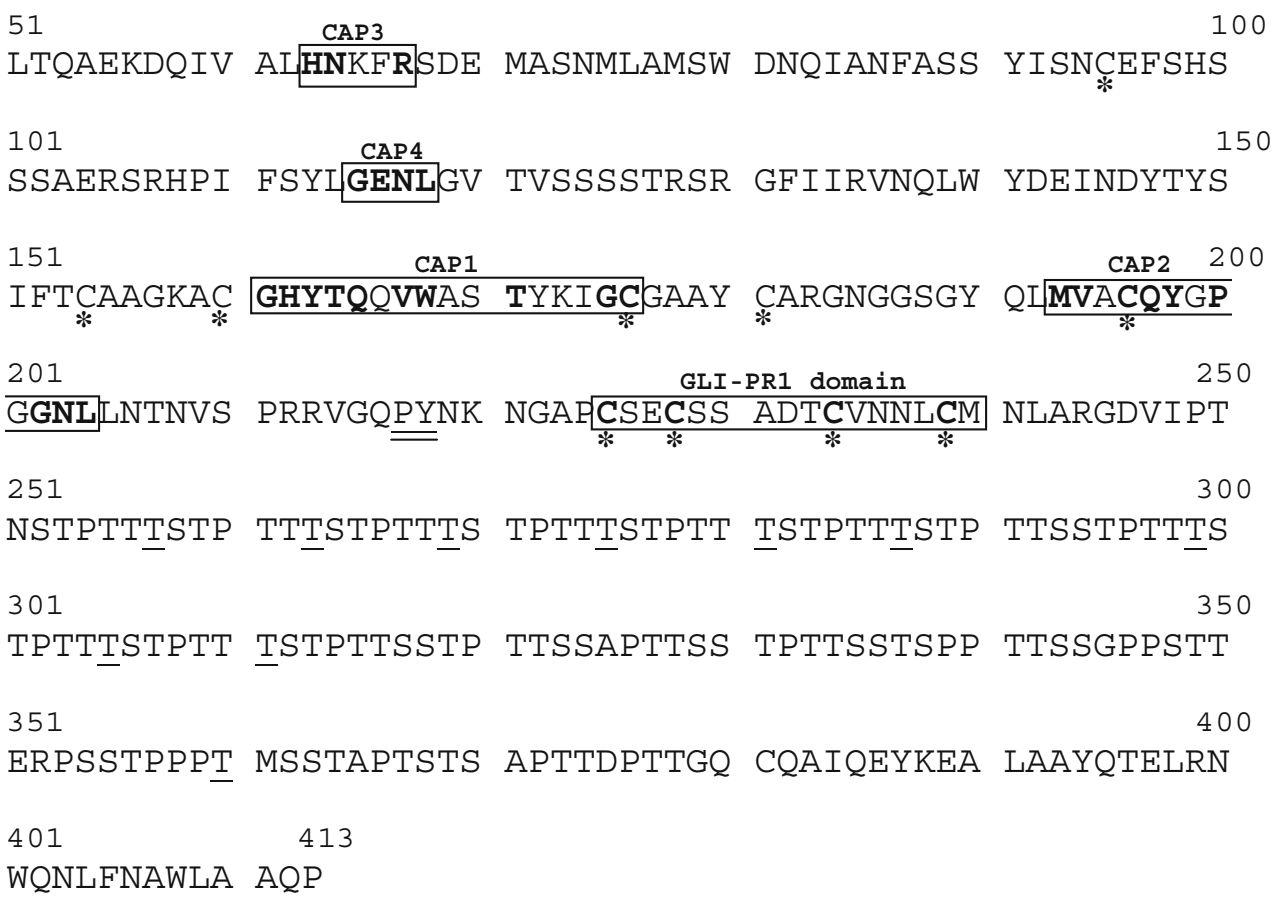

were analyzed and compared with the LPS-injected ascidians.

(74.5\%) and identity (53.8\%) to the collagen-binding adhesin of S. mutans (B9A889; Fig. 3b). The CiCAP amino-acid sequence was aligned with the vertebrate and invertebrate members of CAP-superfamily proteins (CRISP, CRLD, CAPLD, GLIPR1, GLIPR2, PI15 and PI16). Figure 4 shows the phylogenetic tree constructed by the neighbor-joining method. Four main clusters were found. The first includes PI15, CAPLD2, and CRLD2 vertebrate proteins, the second contains invertebrate Ag5 and vertebrate PI16 proteins, the third includes CiCAP and the GLIPR1 human protein, and the last includes the CRISP vertebrate proteins. CiCAP appears to be most closely related to the human GLIPR1 protein.

Quantitative mRNA expression analysis by real-time PCR

Quantitative mRNA expression was examined, by real-time PCR analysis, in the pharynx excised from ascidians after LPS inoculation. In the inflamed pharynx, the expression pattern, compared with that of a housekeeping gene, showed an enhanced level in CiCAP mRNA expression after stimulation. At each time point $(0,1,2,4,8,12,24$, $48 \mathrm{~h}$ ), the gene expression was evaluated in the pharynx from four ascidians. The LPS challenge up-regulated the gene activity, and the CiCAP mRNA level appeared to be increased at 4 and $8 \mathrm{~h}$ post-injection, reaching the highest peak at $8 \mathrm{~h}$; expression decreased at $12 \mathrm{~h}$ and further increased at $24 \mathrm{~h}$ (Fig. 5). As a control for the inducing effect of LPS, the same number of MS-injected ascidians At $8 \mathrm{~h}$ and $24 \mathrm{~h}$ post-injection of MS, when the gene expression attributable to LPS treatment reached a peak, a significantly lower activation was detected (see Fig. 5).

ISH assay

At 4-8 $\mathrm{h}$ after LPS inoculation, ISH analysis of transverse histological sections of the body wall showed numerous CiCAP-expressing hemocytes in the pharynx bars (Fig. 6a, c). In the corresponding sections from naive ascidians, a few positive haemocytes could be seen (Fig. 6b, d). Stigmata epithelium and associated hemocytes expressed CiCAP mRNA only after LPS activation (Fig. 6e); no expression was observed in untreated animals (Fig. 6f). Under the epidermis lining the tunic, the connective tissue lining the hemolymph lacunae frequently contained CiCAP-expressing cells as an effect of the LPS inoculation, whereas positive cells were rare in naive ascidians (Fig. 6g, h, respectively). In inoculated tissue, positive hemocytes appeared to be amoebocytes (Fig. 6g, inset). Controls with the sensestrand probe were negative, both in tissues and in circulating hemocytes (Fig. 6a-c, g, insets).

\section{Discussion}

The innate immune system is the first line of inducible host defense against bacterial, fungal, and viral pathogens. 
Fig. 3 a Alignment of the amino-acid sequence of the CiCAP of $C$. intestinalis with human glioma pathogenesisrelated 1 (GLIP1, P48060). b Alignment of the amino-acid sequence of the CiCAP of $C$. intestinalis with the collagenbinding adhesin of Streptococcus mutans (STRMU, B9A889). Gray-shaded boxes show CAP motifs, asterisks conserved amino acids within the domains, colons conserved residues, dots chemically similar residues, dashes gaps introduced for maximal alignment a

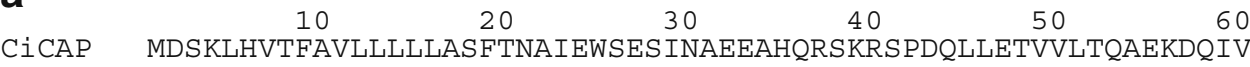

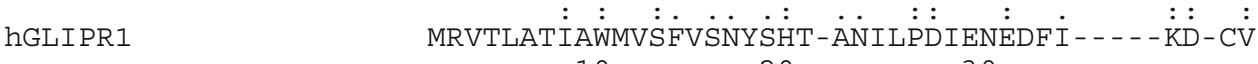
$\begin{array}{lll}10 & 20 & 30\end{array}$

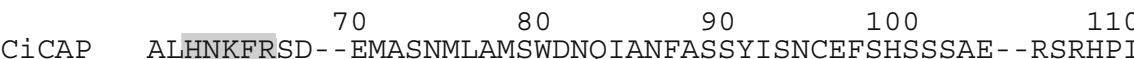

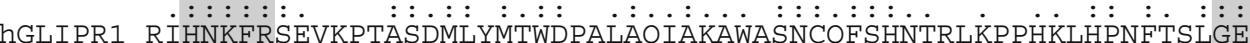

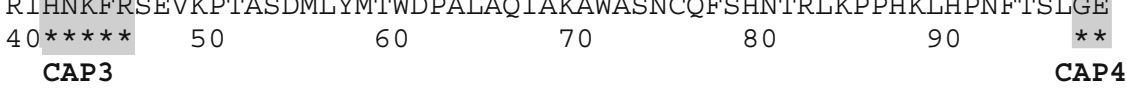

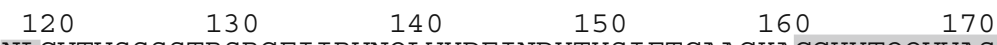

CICAP NLGVTVSSSSTRSRGFI IRVNQLWYDEINDYTYSIFTCAAGKACGHYTQQVWASTYKIGC

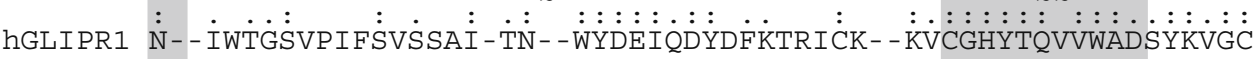

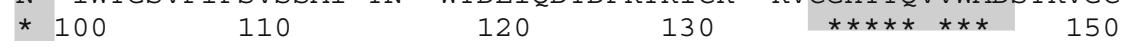
CAP 4 CAP1

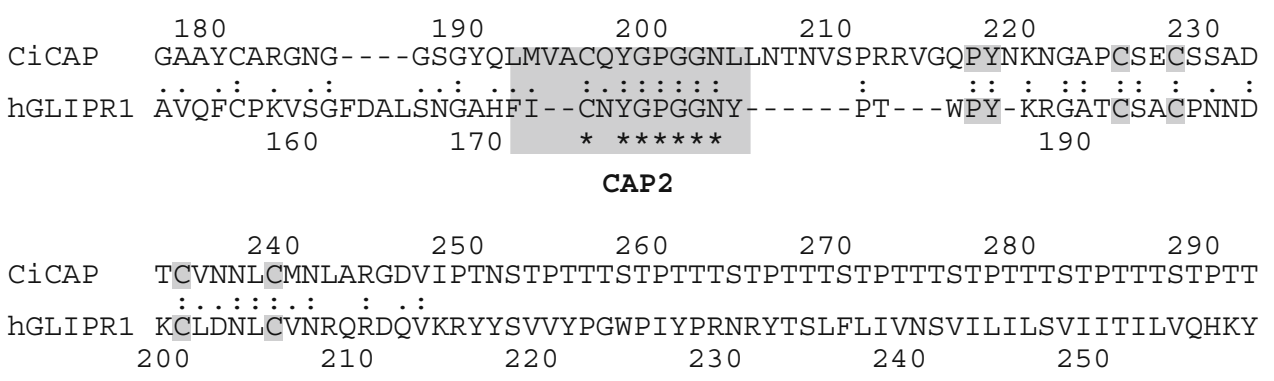

b

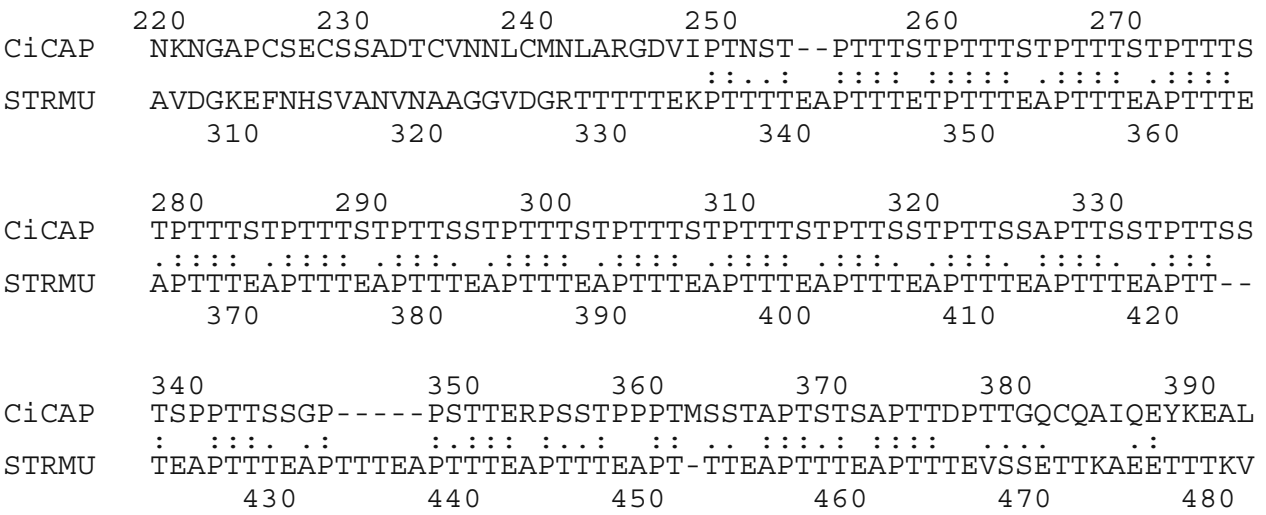

Because both invertebrates and vertebrates respond to substances derived from pathogens, the identification of common patterns of activation of immune genes may shed some light on the action and evolution of the immune system.

CAP proteins represent a widely distributed family of proteins involved in both the innate and adaptive immune responses (Gibbs et al. 2008). Several components of this superfamily have been identified mainly in vertebrates, with few data existing on their role in invertebrates. We have used a subtractive hybridization strategy for selective amplification of differentially expressed sequences allowing the isolation of a CAP transcript from the $C$. intestinalis pharynx after LPS inoculation. This strategy has enabled us to isolate a novel form of a CAP protein (CiCAP) that displays a modular structure with a $\mathrm{NH}_{2}$ terminal region having features in common with other members of the CAP superfamily. In this region, CiCAP displays highly conserved motifs in the CAP domain (CAP1-4), which has been shown to be buried within the core of the tertiary structure of other CAP proteins, and which is probably involved in maintaining the overall structure of the protein (Shikamoto et al. 2005). Furthermore, an additional Cterminal extension of about 160 amino acids, with a significant similarity to the collagen-binding adhesin of $S$. mutans has been identified (Sato et al. 2004). This kind of architecture has not been found in any other protein belonging to the CAP superfamily so far. Domain organization analysis and 


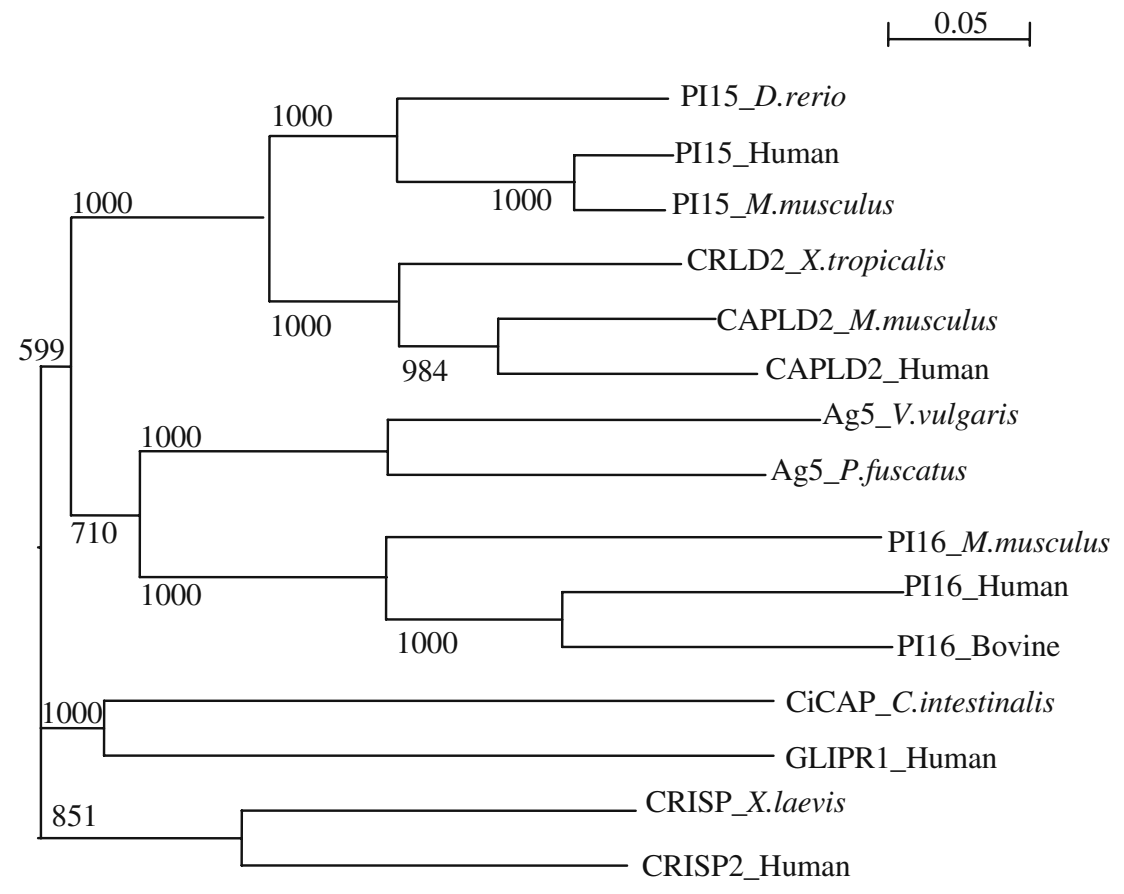

Fig. 4 Phylogenetic tree of deduced amino-acid sequences of CiCAP of $C$. intestinalis and of vertebrate and invertebrate members of CAP superfamily. The tree was constructed by the neighbour-joining method and bootstrap analysis (numbers percentages of 1000 bootstrap replicates in which the same internal branch was recovered). Bar 0.05 (number of amino-acid residues substitutions for site) alignment of the $\mathrm{NH}_{2}$ terminal region of the CiCAP to CAP vertebrate members has shown that this protein originated from a common ancestor gene, and it appears to be related to the vertebrate GLIPR1 proteins. In this respect, the CiCAP protein might represent a case of opposing evolutionary

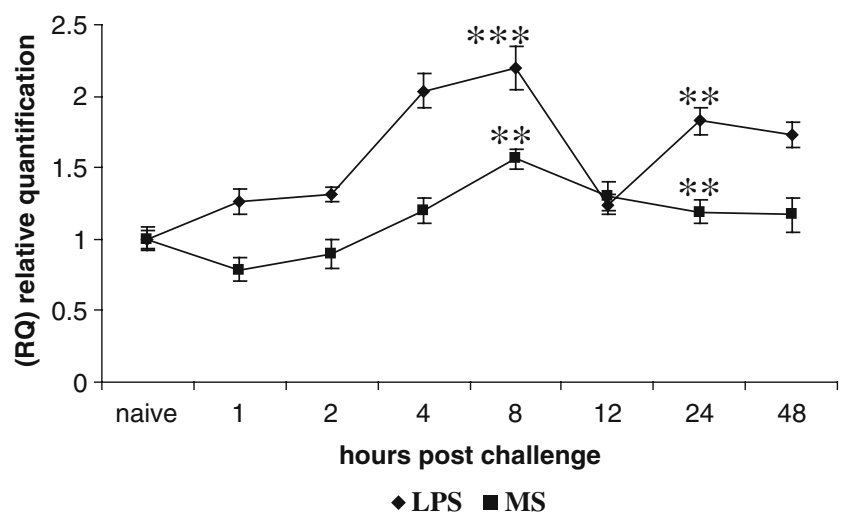

Fig. 5 Quantitative real-time PCR. Relative quantification $(R Q)$ of the induction of the CiCAP gene compared with an internal control. Numbers on the $x$-axis indicate the time after injection of the animal with LPS or marine solution (MS) and RNA sampling. The data on the $y$-axis show the relative increase of the transcriptional activity. Significance was evaluated by comparing the values with the expression level of untreated pharynx from four ascidians. $* * P<0.01, * * * P<0.001$. Multiple comparisons of the time-course profiles were performed by a one-way analysis of variance, and the different groups were compared by the Tukey $t$-test patterns: the first leading to the strong conservation of several sequence motifs probably responsible for the threedimensional structure of the protein, and the second leading to different C-terminal extensions that might justify independent biological functions. Indeed, genome sequencing of many organisms has revealed that species differ in the number and types of possessed genes. New genes arise at various points in evolutionary time, as a result of the juxtaposition of various pre-existing exons in new combinations (Gilbert 1978), by mutational modifications of duplicate genes and retroposition (Long et al. 2003), thereby conferring novel functions enabling organisms to tackle environmental challenges.

The involvement of CiCAP in $C$. intestinalis innate immunity responses has been detected by real-time PCR analysis of the inflamed pharynx. As previously reported, $C$. intestinalis tumor necrosis factor $\alpha(\mathrm{CiTNF} \alpha)$, the type IXlike collagen $\alpha$-chain, collectins, and complement pathway components can be up-regulated following LPS challenge of the pharynx tissues (Parrinello et al. 2008, 2010; Bonura et al. 2009; Vizzini et al. 2008). In this study, we have found that LPS inoculation enhances the expression of CiCAP mRNA. In the pharynx, the CiCAP mRNA level increases in a statistically significant fashion at $4 \mathrm{~h}$ and $8 \mathrm{~h}$, then decreases at $12 \mathrm{~h}$, followed by a further increase at $24 \mathrm{~h}$. A similar time course response characterizes the type IX-like collagen $\alpha$-chain and $C$. intestinalis mannose-binding lectin 
Fig. 6 ISH assay of transverse sections of the body wall of C. intestinalis. Histological sections from ascidians at 4-8 $\mathrm{h}$ after LPS inoculation. a Pharynx bars (phb). Bar $100 \mu \mathrm{m}$. Inset Section treated with sense probe. Bar $50 \mu \mathrm{m}$. c Pharynx bars $(p h b)$ and hemocyte with large granules (hlg). Bar $40 \mu \mathrm{m}$. Inset Hemocyte with large granules treated with sense probe (top) and with anti-sense probe (bottom). Bar $10 \mu \mathrm{m}$. e Stigmata. Bar $40 \mu \mathrm{m}$. g Connective tissue (co) under the epidermis $(e p)$ with lacunae (lac) of the open circulatory system, granular amebocytes $(\mathrm{g} a)$, and muscles $(\mathrm{m})$. Bar $40 \mu \mathrm{m}$. Inset Granular amebocyte treated with sense probe $(t o p)$ and with anti-sense probe (bottom). Bar $10 \mu \mathrm{m}$. Histological sections from naive ascidians. b Pharynx bars ( $p h b)$. Bar $100 \mu \mathrm{m}$. Inset Section treated with sense probe. Bar $50 \mu \mathrm{m}$. d Pharynx bars (phb) and hemocyte with large granules (hlg). Bar $40 \mu \mathrm{m}$. f Stigmata. Bar $40 \mu \mathrm{m}$.

h Connective tissue ( $c o$ ) under the epidermis $(e p)$ with lacunae (lac) of the open circulatory system, granular amebocytes $(\mathrm{ga})$, hemocyte with large granules $(h l g)$, and muscles $(m)$. Bar $40 \mu \mathrm{m}$
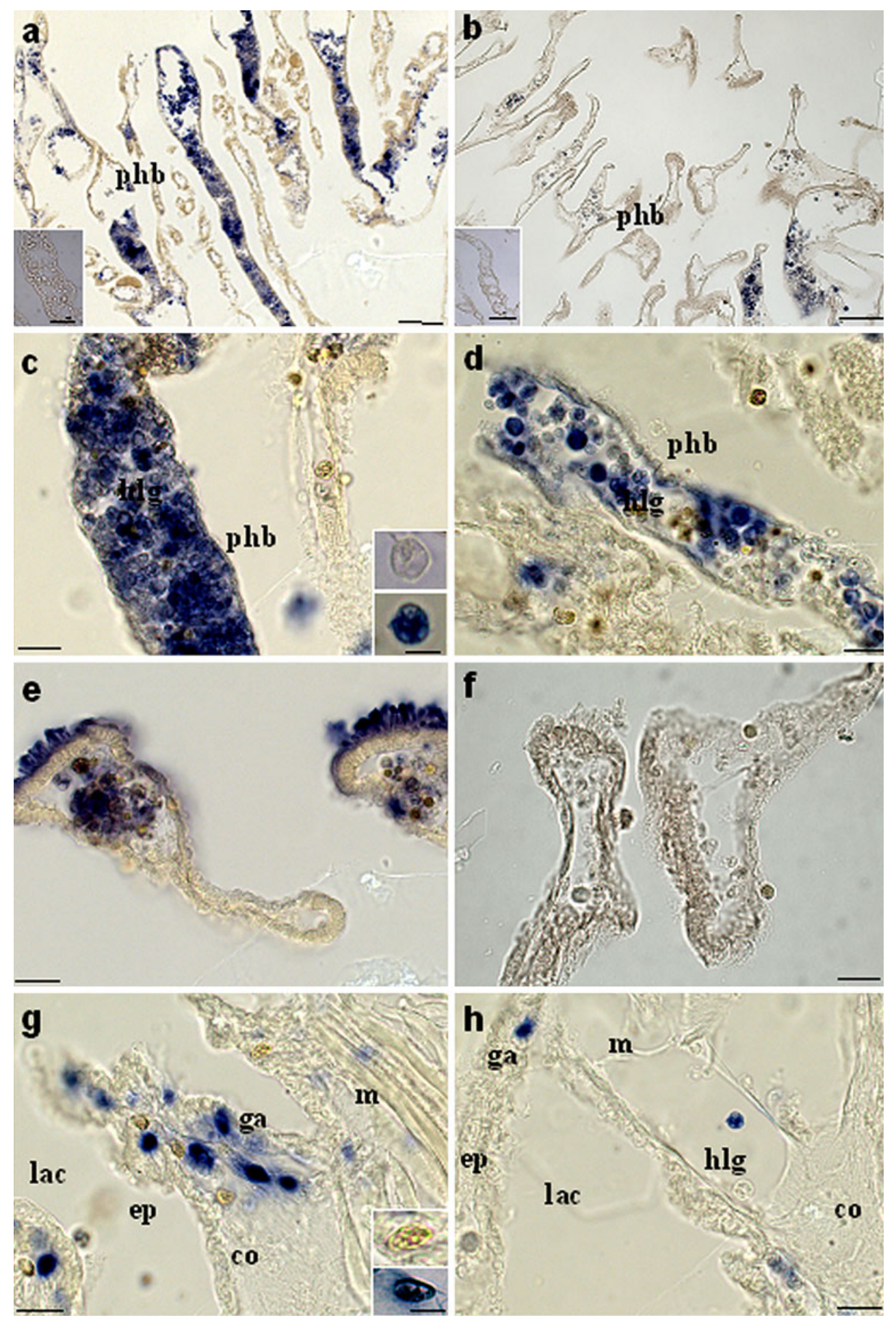

(Bonura et al. 2009; Vizzini et al. 2008). The meaning of the second increase in the expression profiles of inflammatory molecules remains to be clarified. One possible explanation is a delayed inflammatory response by distinct tissues of the pharynx; this might include hemocytes inside the vessels and epithelial tissues. On the other hand, we do not know the regulatory effect of other inflammatory molecules in re- sponse to external signals that might explain the second wave of CiCAP upregulation at $24 \mathrm{~h}$ post-injection. Moreover, we cannot exclude the possibility that the decrease in expression is attributable to cell death after LPS injection, whereas the subsequent increase might be attributable to hemocyte proliferation and the appearance of new CiCAP-expressing circulating cells. 
Based on previously published papers (Bonura et al. 2009; Vizzini et al. 2008), ISH has been performed in order to identify challenged hemocytes inside the pharynx vessels at the time course corresponding to the main expression peak. At $4 \mathrm{~h}$ after inoculation, LPS induces a prompt CiCAP upregulation in hemocytes with large granules contained both in vessels and in associated with connective tissue lining the hemolymph lacunae of the open circulatory system. Although a cell count has not been performed, the CiCAP-expressing hemocytes seem to be more abundant than those found in naive ascidians. Interestingly, the epithelium of pharynx stigmata expresses CiCAP only after LPS challenge. This result is in agreement with a recent paper on CiTNF $\alpha$ expression (Parrinello et al. 2010) disclosing that several regions of the pharynx epithelial tissues are probably involved in the inflammatory response.

In conclusion, we have described the isolation of a novel CAP gene that is up-regulated after LPS injection in the body wall of C.intestinalis. The CiCAP expression profile together with studies on tissue localization suggest a possible role for this protein in the ascidian innate immune responses.

\section{References}

Azumi K, De Santis R, De Tomaso A, Rigoutsos I, Yoshizaki F, Pinto MR, Marino R, Shida K, Ikeda M, Ikeda M, Arai M, Inoue Y, Shimizu T, Satoh N, Rokhsar DS, Du Pasquier L, Kasahara M, Satake M, Nonaka M (2003) Genomic analysis of immunity in a urochordate and the emergence of the vertebrate immune system: "waiting for Godot". Immunogenetics 55:570-581

Bonura A, Vizzini A, Salerno G, Parrinello N, Longo V, Colombo P (2009) Isolation and expression of a novel MBL-like collectin cDNA enhanced by LPS injection in the body wall of the ascidian Ciona intestinalis. Mol Immunol 46:2389-2394

Cammarata M, Benenati G, Odom EW, Salerno G, Vizzini A, Vasta GR, Parrinello N (2007) Isolation and characterization of a fish F-type lectin from gilt head bream (Sparus aurata) serum. Biochim Biophys Acta 1770:150-155

Cammarata M, Arizza V, Cianciolo C, Parrinello D, Vazzana M, Vizzini A, Salerno G, Parrinello N (2008) The prophenoloxidase system is activated during the tunic inflammatory reaction of Ciona intestinalis. Cell Tissue Res 333:481-492

Del Valle A, Jones BF, Harrison LM, Chadderdon RC, Cappello M (2003) Isolation and molecular cloning of a secreted hookworm platelet inhibitor from adult Ancylostoma caninum. Mol Biochem Parasitol 129:167-177

Delsuc F, Brinkmann H, Chourrout D, Philippe H (2006) Tunicates and not cephalochordates are the closest living relatives of vertebrates. Nature 439:965-968

Gibbs GM, Scanlon MJ, Swarbrick J, Curtis S, Gallant E, Dulhunty AF, O'Bryan MK (2006) The cysteine-rich secretory protein domain of Tpx-1 is related to ion channel toxins and regulates ryanodine receptor $\mathrm{Ca}^{2+}$ signaling. J Biol Chem 281:4156-4163

Gibbs GM, Roelants K, O'Bryan MK (2008) The CAP superfamily: cysteine-rich secretory proteins, antigen 5 , and pathogenesisrelated 1 proteins - roles in reproduction, cancer, and immune defense. Endocr Rev 29:865-897
Gibbs GM, Lo JC, Nixon B, Jamsai D, O'Connor AE, Rijal S, Sanchez-Partida LG, Hearn MT, Bianco DM, O'Bryan MK (2010) Glioma pathogenesis-related 1-like 1 is testis enriched, dynamically modified, and redistributed during male germ cell maturation and has a potential role in sperm-oocyte binding. Endocrinology 151:2331-2342

Gilbert W (1978) Why genes in pieces? Nature 271:501

Guo M, Teng M, Niu L, Liu Q, Huang Q, Hao Q (2005) Crystal structure of the cysteine-rich secretory protein stecrisp reveals that the cysteine-rich domain has a $\mathrm{K}^{+}$channel inhibitor-like fold. J Biol Chem 280:12405-12412

Hawdon JM, Hotez PJ (1996) Hookworm: developmental biology of the infectious process. Curr Opin Genet Dev 6:618-623

Kovalick GE, Griffin DL (2005) Characterization of the SCP/TAPS gene family in Drosophila melanogaster. Insect Biochem Mol Biol 35:825-835

Long M, Deutsch M, Wang W, Betran E, Brunet FG, Zhang J (2003) Origin of new genes: evidence from experimental and computational analyses. Genetica 118:171-182

Moyle M, Foster DL, McGrath DE, Brown SM, Laroche Y, De Meutter J, Stanssens P, Bogowitz CA, Fried VA, Ely JA, et al (1994) A hookworm glycoprotein that inhibits neutrophil function is a ligand of the integrin $\mathrm{CD} 11 \mathrm{~b} / \mathrm{CD} 18$. J Biol Chem 269:10008-10015

Parrinello N, Patricolo E, Canicattì C (1984) Inflammatory-like reaction in the tunic of Ciona intestinalis (Tunicata). Encapsulation and tissue injury I. Biol Bull 167:229-37

Parrinello N, De Leo G, Di Bella MA (1990) Fine structural observations of the granulocytes involved in the tunic inflammatory-like reaction of Ciona intestinalis (Tunicata). J Invertebr Pathol 56:181-189

Parrinello N, Vizzini A, Arizza V, Salerno G, Parrinello D, Cammarata M, Giaramita FT, Vazzana M (2008) Enhanced expression of a cloned and sequenced Ciona intestinalis TNFalpha-like (CiTNF alpha) gene during the LPS-induced inflammatory response. Cell Tissue Res 334:305-317

Parrinello N, Vizzini A, Salerno G, Sanfratello MA, Cammarata M, Arizza V, Vazzana M, Parrinello D (2010) Inflamed adult pharynx tissues and swimming larva of Ciona intestinalis share CiTNFalpha-producing cells. Cell Tissue Res 341:299-311

Ren C, Ren CH, Li L, Goltsov AA, Thompson TC (2006) Identification and characterization of RTVP1/GLIPR1-like genes, a novel p53 target gene cluster. Genomics 88:163-172

Sato Y, Okamoto K, Kagami A, Yamamoto Y, Igarashi T, Kizaki H (2004) Streptococcus mutans strains harboring collagen-binding adhesin. J Dent Res 83:534-539

Shikamoto Y, Suto K, Yamazaki Y, Morita T, Mizuno H (2005) Crystal structure of a CRISP family $\mathrm{Ca}^{2+}$-channel blocker derived from snake venom. J Mol Biol 350:735-743

Swalla BJ, Cameron CB, Corley LS, Garey JR (2000) Urochordates are monophyletic within the deuterostomes. Syst Biol 49:52-64

Szyperski T, Fernandez C, Mumenthaler C, Wuthrich K (1998) Structure comparison of human glioma pathogenesis-related protein GliPR and the plant pathogenesis-related protein P14a indicates a functional link between the human immune system and a plant defense system. Proc Natl Acad Sci USA 95:22622266

Thompson JD, Higgins DG, Gibson TJ (1994) CLUSTAL W: improving the sensitivity of progressive multiple sequence alignment through sequence weighting, position-specific gap penalties and weight matrix choice. Nucleic Acids Res 22: $4673-4680$

Trexler M, Banyai L, Patthy L (2000) The LCCL module. Eur J Biochem 267:5751-5757

Urayama S, Harada Y, Nakagawa Y, Ban S, Akasaka M, Kawasaki N, Sawada H (2008) Ascidian sperm glycosylphosphatidylinositol- 
anchored CRISP-like protein as a binding partner for an allorecognizable sperm receptor on the vitelline coat. J Biol Chem 283:21725-21733

Vizzini A, Pergolizzi M, Vazzana M, Salerno G, Di Sano C, Macaluso P, Arizza V, Parrinello D, Cammarata M, Parrinello N (2008) FACIT collagen (1alpha-chain) is expressed by hemocytes and epidermis during the inflammatory response of the ascidian Ciona intestinalis. Dev Comp Immunol 32:682-692

Yamazaki Y, Morita T (2004) Structure and function of snake venom cysteine-rich secretory proteins. Toxicon 44:227-231

Zelensky AN, Gready JE (2005) The C-type lectin-like domain superfamily. FEBS J 272:6179-6217 